\title{
A clinical approach to gynecomastia
}

\author{
L D Ranasinghe ${ }^{1}$, C J Subasinghe ${ }^{1}$, D U S Bulugahapitiya ${ }^{1}$, D T Muthukuda ${ }^{2}$
}

Sri Lanka Journal of Diabetes, Endocrinology and Metabolism 2015; 5: 23-27

\begin{abstract}
Gynecomastia is the commonest breast condition in male, which can cause significant psychological distress and anxiety to the patient. It refers to the benign enlargement of the male breast caused by proliferation of glandular breast tissue due to an imbalance between the inhibitory effect of androgen and stimulatory effect of estrogen. Gynecomastia can be physiological during neonatal period, puberty or old age. Various drugs, systemic disorders, benign or malignant tumours and hypogonadism can also lead to gynecomastia, while about $25 \%$ of cases are idiopathic. Gynecomastia should be differentiated from pseudogynecomastia (adipomastia), which is characterized by excessive accumulation of adipose tissue without glandular proliferation. A detailed history and examination helps to exclude differential diagnosis, identify the underlying etiology and to assess the severity of the disease and the concerns of the patient. The management of gynecomastia depends on the underlying etiology and the concerns of the patient. This may include interventions for relief of pain or discomfort, restoration of normal appearance and reassurance regarding cancer. Treatment options include watchful waiting, pharmacotherapy and surgery.
\end{abstract}

\section{Introduction}

Gynecomastia refers to benign enlargement of the male breast caused by proliferation of glandular breast tissue (1). It is the commonest breast condition affecting males and it sprevalence varies between 32-65\% according to factors like age, lifestyle etc (2). Gynecomastia can cause significant psychological distress and anxiety to the patient and although mostly benign may signal a sinister underlying disease. Therefore proper evaluation and treatment is of paramount importance.

\section{Pathophysiology of gynecomastia}

In both males and females, estrogen causes enlargement of the breast by inducing proliferation and differentiation of ductal and periductal tissues. However in males acinar development usually does not occur due to lack of progesterone (3).

Gynecomastia is caused by an imbalance between the stimulatory effect of free estrogen on growth and differentiation of breast tissue and inhibitory effect of free androgen. An increased estrogen-to-androgen ratio due to absolute increase in estrogen production, relative decrease in androgen production or a combination of both can give rise to gynecomastia (1).

The extent of glandular proliferation depends on the intensity and the duration of stimulation. The early stages (first six months after onset) of gynecomastia are characterized by hyperplasia of ductal and peri ductal tissues with increased stromal fibroblastic proliferation and periductal inflammation leading to pain or tenderness in the breast. The later stages (after a year) are characterized by marked stromal fibrosis without an inflammatory response, thereby causing painless gynaecomastia (4).

\section{Etiology and differential diagnosis of gynecomastia}

Gynecomastia can be physiological or pathological. Physiological gynecomastia shows a trimodal age distribution with first, second and third peaks occurring during infancy, puberty and old age respectively (5). Neonatal gynecomastia occurs in about $60-90 \%$ of boys due to exposure to high concentration of maternal estrogens in utero. It is transient and resolves within a few weeks after birth (1).

Pubertal gynecomastia usually begins at ages of 10-12 years and peaks at ages of 13-14 years. Breast enlargement of more than $0.5 \mathrm{~cm}$ in diameter occurs in about $60-70 \%$ of boys by the age of 14 years. It is attributed to a transient more rapid rise of serum estrogen relative to testosterone during early puberty. It usually regresses within 1-2 years with pubertal progression and rise in testosterone levels.

Senile gynecomastia occurs in $24-65 \%$ of men at 50 to 80 years of age and is thought to be due to increased adiposity with aging (1). Androgens are converted into

${ }^{1}$ Department of diabetes and endocrinology, Colombo South teaching hospital, ${ }^{2}$ Diabetes and endocrine unit, provincial general hospital, Ratnapura. 
estrogens in adipose tissue by aromatase and age-related increase in aromatase activity result in higher estrogen production rates in older men.

Pathological gynecomastia may result from the excessive estrogen, deficient androgens or androgen resistance (Table 1). Various drugs (Table 2), systemic disorders, benign or malignant tumors and hypogonadism leads to gynecomastia. About $25 \%$ of cases are idiopathic. Environmental contamination with xenoestrogens or estrogen-like substances and abuse of anabolic steroids are increasingly recognized causes.

Gynecomastia should be differentiated from pseudogynecomastia (adipomastia) which is characterized by excessive accumulation of adipose tissue without glandular proliferation. Presence of any atypical features like unilateral enlargement, skin changes, hard consistency and nipple discharge should trigger evaluation for breast cancer.

\section{Clinical evaluation}

A detailed history and examination helps to exclude differential diagnosis, identify the underlying etiology and to assess the severity of the disease and the concerns of the patient.

History should include the onset and duration of breast enlargement, nipple discharge, retraction, symptoms of pain or tenderness, weight loss or gain, medication history, the presence of systemic illness, fertility, sexual function. Family history of gynecomastia may suggest androgen insensitivity syndrome or familial aromatase excess (8).
Table 1. Causes of gynecomastia (6)

\section{Physiologic causes}

Neonatal, pubertal, involutional (senile)

\section{Systemic disorders}

Organ failure: Hepatic cirrhosis, chronic kidney disease

Endocrine disorders: Hyperthyroidism, Cushing syndrome, acromegaly

\section{Estrogen excess}

Estrogens or estrogen receptor agonists: Marijuana, smoke, digoxine, testosterone

Increased peripheral aromatase activity: Obesity, aging, familial

Estrogen secreting tumors: Leydig/Sertoli cell tumor, adrenal carcinoma

hCG secreting tumors: Germ cell, lung, hepatic carcinoma

\section{Androgen deficiency or resistence}

Androgen deficiency: Primary or secondary hypogonadism, hyperprolactinaemia

Androgen resistence: Congenital or acquired androgen resistance, drugs eg. spironolactone

\section{Idiopathic causes}

Table 2. Drugs associated with gynecomastia (7)

Hormones

Antiandrogens/inhibitors of androgen synthesis

Antibiotics

Antiulcer medications

Chemotherapeutic agents

Cardiovascular drugs

Psychoactive drugs

Miscellaneous
Androgens, anabolic steroids, estrogens, estrogen agonists and $\mathrm{hCG}^{\mathrm{a}}$

Flutamide, nilutamide, cyproterone, GnRH ${ }^{\mathrm{a}}$ agonists

Ketoconazole ${ }^{\mathrm{b}}$, metronidazole, isoniazid

Omeprazole, ranitidine, cimetidine ${ }^{\mathrm{b}}$

Methotrexate, alkylating agents

Digoxin ${ }^{\mathrm{b}}, \mathrm{ACEI}^{\mathrm{a}}, \mathrm{CCB}^{\mathrm{a}}$, amiodaronespironolactone, methyldopa

Tricyclic antidepressants, haloperidol and atypical anti psychotic agents, diazepam

Metoclopramide, phenytoin, theophylline, anti retroviral therapy

${ }^{a}$ ACEI - angiotensin converting enzyme inhibitor; CCB - calcium channel blocker; GnRH - gonadotropin releasing hormone; hCG - human chorionic gonadotropin

${ }^{\mathrm{b}}$ Denotes stronger association 
Figure 1. Differentiation of gynecomastia from pseudogynecomastia (7)

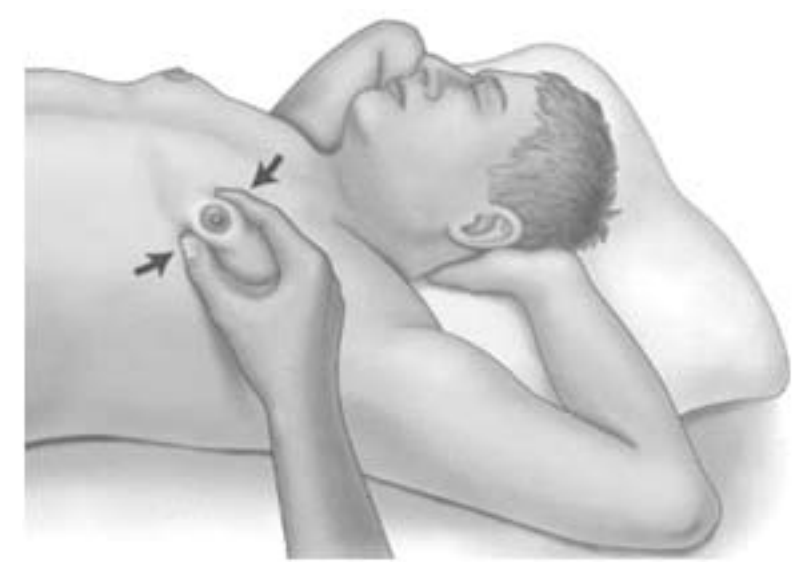

Figure 2. Diagnostic algorithm for gynecomastia (11)

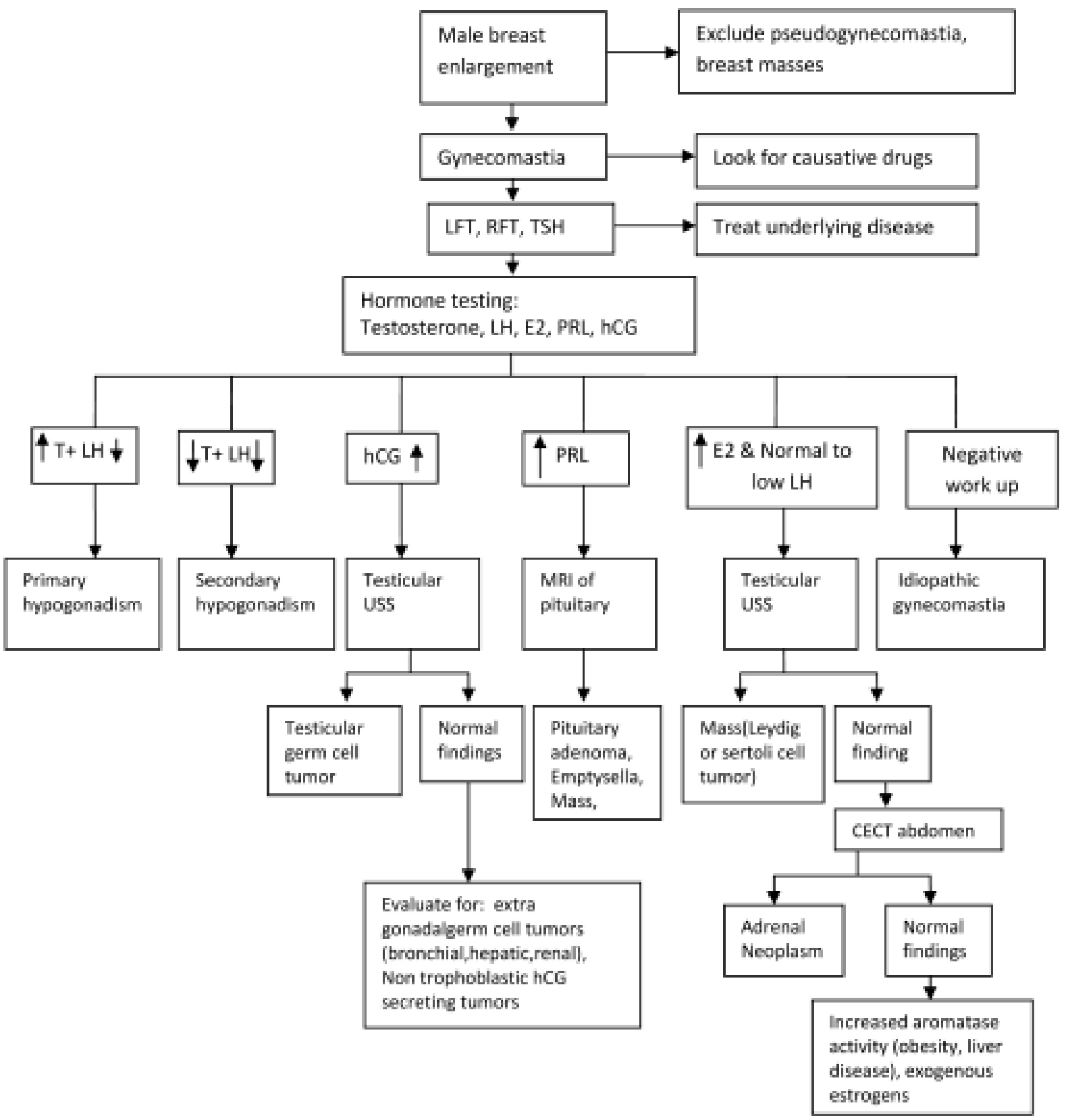


Gynecomastia is identified clinically as a generalized enlargement of male breast with palpable glandular tissue. The later is firm or rubbery and contains fibrous-like cords. The breast should be examined when the patient lies on his back with his hands behind his head. The examiner should place her/his thumb and forefinger on each side of the breast and slowly bring them together (Figure 1). In true cases of gynecomastia, the examiner will feel a rubbery to firm disc of tissue that is concentric with the nippleareolar complex and freely mobile. In contrast, in pseudogynecomastia the fingers will not meet any resistance until they reach the nipple (9).

\section{Biochemical evaluation}

Biochemical evaluation should be considered in the cases of true gynecomastia without clear explanation. Patients with physiologic gynecomastia do not require further evaluation. Patients with breast enlargement that is large (glandular tissue $>5 \mathrm{~cm}$ in obese men, $>2 \mathrm{~cm}$ in lean men), recent and rapid in onset, progressive, symptomatic or suspicious for malignancy need further investigations (6). Common medical conditions like renal, liver and thyroid disease should be excluded by appropriate investigations. Hormonal evaluation including measurement of levels of total testosterone, Luteinizing Hormone (LH), estradiol, prolactin and hCG may reveal an underlying pituitary, gonadal, and extragonadal pathologies including neoplasms. Measurement of bioavailable testosterone may be considered in patients with marginally low testosterone or those who have conditions causing altered SHBG level. A stepwise approach for bio chemical evaluation is shown in algorithm depicted in the (Figure 2). Idiopathic gynecomastia is diagnosed when all the biochemical testing is negative.

Mammography can be used to differentiate benign and malignant male breast diseases fairly accurately (10).

\section{Management of gynecomastia}

The management of gynecomastia depends on the underlying etiology and the concerns of the patient. This may include interventions for relief of pain or discomfort, restoration of normal appearance and reassurance regarding cancer.

\section{Watchful waiting}

Gynecomastia of recent onset (less than six months) often regresses spontaneously in vast majority of patients and symptoms like pain, tenderness tend to resolve with time as the inflammation settles and fibrosis occurs (12). Therefore observation alone with periodical follow up (once in 6 months) would be sufficient for most adolescents, and also for most men without considerable underlying pathology according to appropriate workup after stopping offending medications and/or treating any underlying disorders. Pubertal gynecomastia regresses in 85 to 90 percent within six months to two years. However if gynecomastia is associated with severe breast enlargement, pain, tenderness, and embarrassment that interferes with the patient's normal daily activities treatment should be initiated. Treatments may also be needed to the patients with persistent gynecomastia which is lasting more than a year. The degree of breast enlargement and symptoms that trigger treatment are dependent upon the perception of the patient.

\section{Pharmacotherapy}

Pharmacotherapy is likely to be effective usually at early stage of the disease (within a year) where there is predominant inflammation and less fibrosis. Several medications can be used to modify hormonal changes involved in gynecomastia. Anti-estrogens, aromatase inhibitors and androgens are the available options. However the data on efficacy and safety of these drugs comes from small studies.

Although clinical trial data are limited, the selective estrogen receptor modulators (SERMs) appear to be effective and generally well-tolerated. It may rapidly reduce the pain and should be considered as first-line therapy for symptomatic cases of acute gynecomastia, or those who have persistent symptoms. However, complete breast regression is usually not achieved with this approach. Raloxifene, tamoxifen and clomiphine citrate are the available options. The latter has limited and variable effect. Lawrence, et al has shown that raloxifene is more effective than tamoxifen in reducing persistent pubertal gynecomastia (13). SERM therapy is usually used for pubertal boys with severe gynecomastia, which is often associated with tenderness. However, the degree of breast enlargement and symptoms that trigger the treatments are dependent upon the perception of boys and their parents. The commonly used doses of SERM are raloxifene $60 \mathrm{mg} /$ day and tamoxifen 10-20 mg/ day.

Tamoxifen has been shown to be effective in reducing breast size and tenderness in adult men. A brief trial (three to six months) of tamoxifen (10 mg twice daily) is recommended for relief of tenderness. The regression of gynecomastia is always partial (14). There is inadequate experience with raloxifene in adult men. In males with hypogonadism, testosterone replacement often improves gynecomastia (15).

Androgens like dihydrotestosterone, danazol, clomiphene, and aromatase inhibitors such as testolactone and anastrozole may also be effective but has limited data and less commonly used (16).

\section{Surgery}

Surgery should be considered for patients whose 
gynecomastia does not regress spontaneously or with medical therapy and causes considerable discomfort and or psychological distress.

Surgery should be considered in men with persistent gynecomastia ( more than1-2 years) since the breast tissue has probably become fibrotic and unresponsive to medical therapy. The Surgery should be delayed in adolescents until the puberty is complete and testis has reached adult size because otherwise the breast tissue may re-grow.

Reduction mammoplasty can be performed using peri areolar or transareolar approach. Skin resection may be necessary for more advanced cases. The surgery can be combined with liposuction in cases with significant amount of fat tissue (14). The potential surgical complications include breast asymmetry, nipple necrosis or flattening, hypertrophic scars, contour irregularity, hematomas, numbness of the nipple and areolar areas. The less invasive techniques (endoscopic subcutaneous mastec-tomy) offer minimal surgical incision and lesser complica-tion rates. Histological analysis of the glandular breast tissue is recommended since an unexpected finding such as spindlecell hemangioendothelioma and papilloma may occur in about $3 \%$ of cases (17).

\section{References}

1. Johnson RE, Murad MH. Gynecomastia: Pathophysiology, evaluation and management. Mayo Clin Proc 2009; 84: 1010-5.

2. Rahmani S, Turton P, Shaaban A, Dall B. Overview of gynecomastia in the modern era and the Leeds gynaecomastia investigation algorithm. Breast J. 2011; 17: 246-55.

3. Wilson JD, Aiman J, MacDonald PC. The pathogenesis of gynecomastia. Adv Intern Med 1980; 25: 1-32.

4. Bembo SA, Carlson HE. Gynecomastia: Its features, and when and how to treat it. Cleve Clin J Med 2004; 71: 511-7.

5. Gikas P, Mokbel K. Management of gynaecomastia: an update. Int J Clin Pract 2007; 61: 1209-15.

6. Shlomo Melmed, Kenneth S. Polonsky, P. Reed Larsen, Henry M. Kronenberg. Testicular disorders. Williams text book of endocrinology. 12th ed. 2011. 719-20.

7. Braunstein GD. Gynecomastia. N Engl J Med 2007; 357(12): 1229-37.

8. Carlson HE. Approach to the patient with gynecomastia. $J$ Clin Endocrinol Metab 2011; 96: 15-21.

9. Barros AC, Sampaio Mde C. Gynecomastia: Physiopathology, evaluation and treatment. Sao Paulo Med J 2012; 130: 187-97.

10. Evans GF, Anthony T, Turnage RH, et al. The diagnostic accuracy of mammography in the evaluation of male breast disease. Am J Surg 2001; 181: 96-100.

11. Braunstein GD. Gynecomastia. N Engl J Med. 1993; 328(7): 490-5.

12. Ruth E. Johnson, M. Hassan Murad. Gynecomastia: apthophysiology, evaluation, and management. Mayo Clin Proc 2009; 84(11): 1010-5.

13. Lawrence SE, Faught KA, Vethamuthu J, Lawson ML. Beneficial effects of raloxifene and tamoxifen in the treatment of pubertal gynecomastia. J Pediatr 2004; 145(1): 71-6.

14. McDermott MT, Hofeldt FD, Kidd GS. Tamoxifen therapy for painful idiopathic gynecomastia. South Med J 1990; 83: 1283.

15. Gruntmanis U, Braunstein GD. Treatment of gynecomastia. Curr Opin Investig Drugs 2001; 2: 643.

16. Nordt CA, DiVasta AD. Gynecomastia in adolescents. Curr Opin Pediatr 2008; 20(4): 375-82.

17. Handschin AE, Bietry D, Hüsler R, Banic A, Constantinescu M. Surgical management of gynecomastia - a 10-year analysis. World J Surg 2008; 32(1): 38-44. 